\title{
Inferring the Brain Connectivity from MR Diffusion Tensor Data
}

\author{
C. Poupon ${ }^{1,2}$, C.A. Clark ${ }^{1}$, V. Frouin ${ }^{1}$, D. LeBihan ${ }^{1}$, I. Bloch ${ }^{2}$, and \\ J.-F. Mangin ${ }^{1}$ \\ 1 SHFJ, CEA, 91401 Orsay, France \\ cpoupon@shfj.cea.fr \\ http://www-dsv.cea.fr/ \\ 2 ENST, 75013 Paris, France
}

\begin{abstract}
Magnetic resonance diffusion tensor imaging (DTI) has become an established research tool for the investigation of tissue structure and orientation. In this paper, we are interested in the connectivity induced by white matter fibers in the living human brain. Considering the data resolution, this question has to be addressed at the level of fascicles made up by a bunch of parallel fibers. We propose first an algorithm dedicated to the fascicle tracking in a direction map inferred from diffusion data. This algorithm takes into account the usual fan shaped merge of several fascicles in one larger bundle. Then, we propose a way of inferring a regularized direction map from diffusion data in order to get a robust tracking. The regularization stems from a priori knowledge on the white matter geometry embedded in a model of the bending energy of an equivalent spaghetti plate. Finally, we propose a study of the tracking behaviour according to the weight given to the regularization and some examples of the tracking results with real data.
\end{abstract}

\section{Introduction}

Up to now, information on brain anatomical connectivity has only been obtained from post mortem studies. Many anatomical methods have been designed for this purpose. Among all possible approaches, chemical tracers which are transported by the axons from a point of injection either anterogradely or retrogradely, have become the method of choice [1]. These methods have yielded detailed brain connectivity maps in different species like the cat and the monkey. However, major disadvantages of these techniques are the required animal sacrifice and the cumbersome 3D reconstruction of the serial sections. Therefore, there has been increasing interest in the development of MRI-based in vivo tract tracing tools. One direction of research relies on magnetic resonance diffusion imaging for the in vivo study of the human brain.

Calculation and subsequent diagonalization of the water apparent diffusion tensor yields its eigenvalues and eigenvectors. The eigenvector corresponding to the largest eigenvalue may be considered to represent the main direction of diffusion in a voxel. Given that one may ascribe diffusion anisotropy in white matter to a greater hinderence or restriction to diffusion across the fiber axes than along them, the principal eigenvector may be considered to point along the direction 
of a putative fiber bundle crossing this voxel. Considering the orientation of the principal eigenvector in neighbouring voxels, it may be deduced whether the fibers in those voxels are connected or not. While this has been used to segment large white matter structures of the brain such as the corpus callosum and pyramidal tract [2], it is necessary to refine this methodology in order to assert which cortical areas or grey nuclei are connected by fascicles embeded in white matter bundles. The first part of this paper describes a fascicle tracking algorithm dealing with fascicle junctions. This algorithm input is a direction map made up for instance by the tensor first eigenvector. Then we propose a model to compute a regularized direction map from the tensor data in order to improve the robustness of the tracking algorithm. Results with real tensor data show that this model improves the consistency of the tracking.

\section{Methods}

DTI was performed on a 1.5T Signa Echospeed MRI system (General Electric, Milwaukee). Image parameters were: image matrix $=128 \times 128$ pixels, 56 slices, in plane resolution $=1.875 \mathrm{~mm}$, slice thickness $=2.8 \mathrm{~mm}$. A standard T1-weighted image was also acquired.

A first step of the tracking approach consists in defining a volume of interest (VOI) on which will be restricted all the methods described in the following. This VOI, which is made up by the voxels belonging to white matter, is automatically extracted from the T1-weighted image using an algorithm developed in our institution [3]. A morphological dilation is then applied to this segmentation in order to be robust to potential distortions between the echo-planar diffusion images and the high resolution T1-weighted image.

\subsection{A Simple Fascicle Tracking Algorithm}

The diffusion tensor and subsequently the eigen system are calculated for each voxel of the VOI [4]. Then, different approaches can be chosen to try to infer the brain connectivity from the DT data. The simplest idea consists in defining a connectivity rule between adjacent white matter voxels in order to define the major bundles as different connected components [2]. This approach can not really deal with brain connectivity because a bunch of bundles bound to cross the same white matter bottleneck (corpus callosum, internal capsule) belong to the same connected component. In this paper, we develop a different idea which consists in partitioning the white matter domain in a set of putative fascicles represented by chains of linked voxels. Using this approach, the largest bundles are split into a set of fascicles which allows for instance the study of the connectivity induced by the fibers of the corpus callosum. This idea could be related to the notion of tractography introduced by Basser [5].

The partitioning of white matter in a set of fascicles relies on a simple rule to link voxels. This rule is defined from a direction map, namely an image of unitary vectors. In a first approach, this direction map is simply the image of the eigen vector of the diffusion tensor associated with the largest eigenvalue. Let $\boldsymbol{d}(M)$ denotes the direction of voxel $M$. Each voxel $M$ is endowed with a 


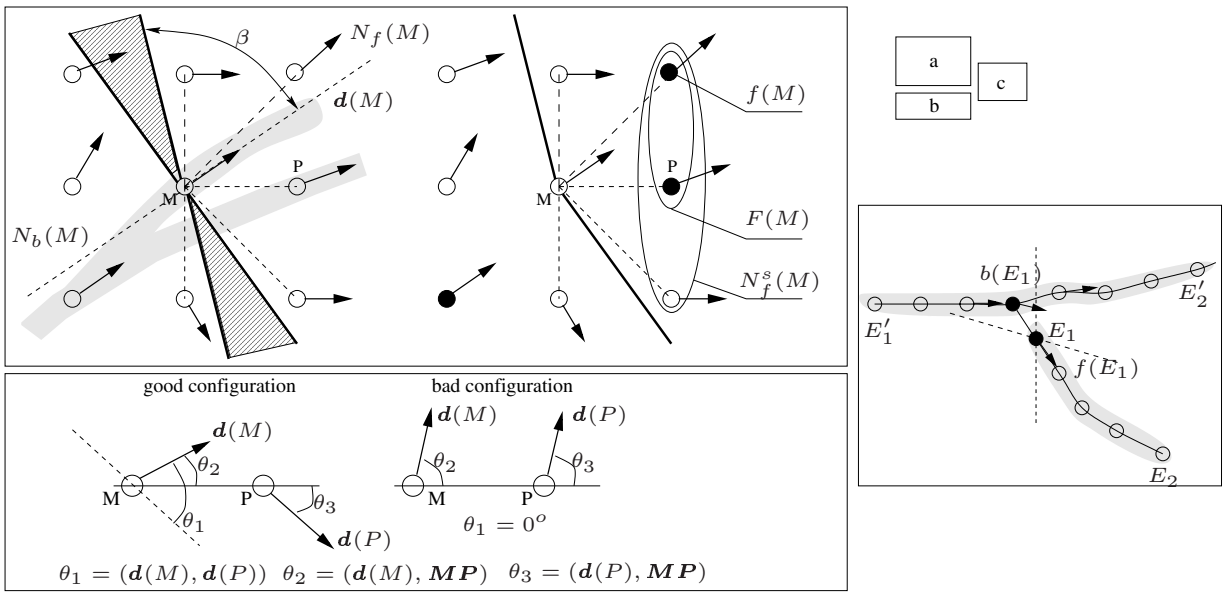

Fig. 1. (a) : construction of the extended neighborhoods of a site M (see text); some underlying fascicles are represented in gray; (b) : the 3 angles used to compute the low curvature criterion $s(M, P)$ (cf. Eq. 1); (c) : fan shaped fascicle split leading to the definition of junctions.

forward and a backward conic neighborhood $\mathcal{N}_{f}(M)$ and $\mathcal{N}_{b}(M)$ defined from $\boldsymbol{d}(M)$ (see Fig. 1a). The conic neighborhood $\mathcal{N}_{f}(M)$ (respectively $\mathcal{N}_{b}(M)$ ) is defined as the subset of $M$ 26-neighbors belonging to the half-cone whose apex is $M$, whose direction is $\boldsymbol{d}(M)$ (resp. $-\boldsymbol{d}(M)$ ) and whose aperture angle is $\beta$ (typically set to $45^{\circ}$ ). A voxel $M$ can be linked to at most one voxel $f(M)$ (resp. $b(M)$ ) of $\mathcal{N}_{f}(M)$ (resp. $\mathcal{N}_{b}(M)$ ). The linked forward neighbor $f(M)$ is defined if the set $\mathcal{N}_{f}^{s}(M)=\left\{P \in \mathcal{N}_{f}(M), M \in \mathcal{N}_{f}(P) \cup \mathcal{N}_{b}(P)\right\}$ is non empty. Simply speaking, this constraint is related to the fact that a consistent connectivity relationship has to be symmetrical. If we define the function (see Fig. 1b):

$$
s(M, P)=\frac{\max ^{2}\left(\left(\boldsymbol{d}(M), \boldsymbol{u}_{M P}\right),\left(\boldsymbol{d}(P) \cdot \boldsymbol{u}_{M P}\right),(\boldsymbol{d}(M) \cdot \boldsymbol{d}(P))\right)}{\|\boldsymbol{M P}\|},
$$

where $\boldsymbol{u}_{M P}=\frac{\boldsymbol{M P}}{\|\boldsymbol{M P}\|}$ and $(\boldsymbol{u}, \boldsymbol{v})$ denotes the angle between directions $\boldsymbol{u}$ and $\boldsymbol{v}$, then the definition of $f(M)$ is (see Fig. 1a):

$$
f(M)=\operatorname{Arg} \min _{P \in \mathcal{N}_{f}^{s}(M)} s(M, P)
$$

Hence, $f(M)$ is the best forward neighbor according to a criterion taking into account the three angles between $\boldsymbol{d}(M), \boldsymbol{d}(P)$ and the direction $\boldsymbol{M} \boldsymbol{P}$ (see Fig. 1). This criterion endows the locally tracked fascicle with the lowest possible curvature. The best backward neighbor $b(M)$ is defined in the same way:

$$
b(M)=\operatorname{Arg} \min _{P \in \mathcal{N}_{b}^{s}(M)} s(M, P),
$$

where $\mathcal{N}_{b}^{s}(M)=\left\{P \in \mathcal{N}_{b}(M), M \in \mathcal{N}_{f}(P) \cup \mathcal{N}_{b}(P)\right\}$ has to be non empty.

The partition of white matter in fascicles stems from the definition of a connectivity rule $\mathcal{C}$ between adjacent voxels. Two voxels $M_{1}$ and $M_{2}$ are connected 
$\left(M_{1} \mathcal{C} M_{2}\right)$ if and only if they are symetrically best neighbors to each other: $\left(M_{1}=f\left(M_{2}\right)\right.$ or $\left.M_{1}=b\left(M_{2}\right)\right)$ and $\left(M_{2}=f\left(M_{1}\right)\right.$ or $\left.M_{2}=b\left(M_{1}\right)\right)$. The fascicles are the connected components defined by this connectivity rule. Since a voxel can not be endowed with more than two neighbors with this rule, simple topological considerations lead to the fact that each fascicle can be represented by a chain of voxels $\left(M_{1}, M_{2}, \ldots, M_{n}\right)$, where $M_{i} \mathcal{C} M_{i+1}$ for $i \in\{1,2, \ldots, n-1\}$. In fact two different kinds of chains can exist. The simple chains whose extremities $M_{1}$ and $M_{n}$ are respectively endowed with only one neighbor for the rule $\mathcal{C}$ correspond to the natural anatomical notion of fascicle. In the following such chains will be denoted unambigously by their extremity pair $\left\{M_{1}, M_{n}\right\}$. The second kind of potential chains corresponds to the closed loops without real extremities for which $M_{n} \mathcal{C} M_{1}$. Such pathological chains should not exist from an anatomical point of view.

\subsection{Towards Brain Connectivity}

The partition of white matter in such mathematically defined fascicles is not sufficient to get access to the brain connectivity level. Indeed, we have to deal with the configurations where a fascicle extremity $E_{1}$ is not located on the boundary but inside white matter, which is a nonsense from an anatomical point of view. Let us assume without loss of generality that the extremity voxel $E_{1}$ is linked to its fascicle $\left\{E_{1}, E_{2}\right\}$ by $f\left(E_{1}\right)\left(f\left(E_{1}\right) \in\left\{E_{1}, E_{2}\right\}\right)$ (see Fig. 1c). Two different cases can occur.

1. If $b\left(E_{1}\right)$ exists, $b\left(E_{1}\right)$ belongs to another fascicle $\left\{E_{1}^{\prime}, E_{2}^{\prime}\right\}$. This configuration can be interpreted anatomically as a "fascicle merge". In the case of two parallel fascicles, such a merge can be related to a decrease of the diameter of the underlying bundle implying a higher fiber density. When the two fascicles follow relatively different directions, the merge occurs at a location where the underlying bundle is split in a fan shaped fashion. In order to deal with this fascicle merge notion, we have to extend the initial linking rule $\mathcal{C}$ to allow the existence of junction voxels. This extension consists in the introduction of two sets of linked forward and backward neighbors, respectively $\mathcal{F}(M)$ and $\mathcal{B}(M)$, for each voxel $M$. For the simple voxels, $\mathcal{F}(M)=\{f(M)\}$ and $\mathcal{B}(M)=\{b(M)\}$. For the junctions, these sets can include more voxels. Let us go back to the configuration introduced above. Since $b\left(E_{1}\right) \in \mathcal{N}_{b}^{s}\left(E_{1}\right)$, either $E_{1} \in \mathcal{N}_{f}\left(b\left(E_{1}\right)\right)$ or $E_{1} \in \mathcal{N}_{b}\left(b\left(E_{1}\right)\right)$. The first case naturally leads to the addition of $E_{1}$ in $\mathcal{F}\left(E_{1}\right)$, the second case leads to the addition of $E_{1}$ in $\mathcal{B}\left(E_{1}\right)$.

2. If $b\left(E_{1}\right)$ does not exist, we face a very unplausible anatomical situation which should be rare but manageable in the future. This situation does not lead to linked neighbor sets extension.

The above considerations have led to the design of an extended set of linked neighbors $\mathcal{F}(M)$ and $\mathcal{B}(M)$. It should be noted that the construction of these neighbor sets does not require the computation of the fascicles which have been mainly introduced for the sake of clarity: 


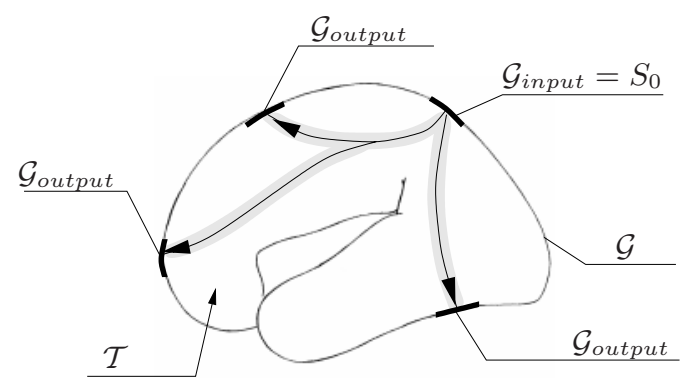

Fig. 2. tracking process; the tracking starts at a subset $\mathcal{G}_{\text {input }}$ of the boundary $\mathcal{G}$ between white and gray matter; it uses $\mathcal{F}(M)$ and $\mathcal{B}(M)$ sets to propagate through white matter $\mathcal{T}$ until a cortical or basal $\mathcal{G}_{\text {output }}$ region is reached.

- if $\mathcal{N}_{f}^{s}(M) \neq \emptyset$ :

$$
\mathcal{F}(M)=\{f(M)\} \cup\left\{P \in \mathcal{N}_{f}^{s}(M) \mid f(P)=M \text { or } b(P)=M\right\},
$$

otherwise $\mathcal{F}(M)=\emptyset$;

- if $\mathcal{N}_{b}^{s}(M) \neq \emptyset$ :

$$
\mathcal{B}(M)=\{b(M)\} \cup\left\{P \in \mathcal{N}_{b}^{s}(M) \mid f(P)=M \text { or } b(P)=M\right\},
$$

otherwise $\mathcal{B}(M)=\emptyset$.

With these definitions we are now able to define clearly the way of inferring the brain connectivity through tracking.

The VOI related to white matter is made up by three kinds of voxels. Let $\mathcal{G}$ denote the set of voxels located at the boundary of the VOI (see Fig. 2). A simple point of view leads us to consider this set as the gate to gray matter. Let $\mathcal{T}$ denote a second set of voxels which verify $\mathcal{F}(M) \neq \emptyset$ and $\mathcal{B}(M) \neq \emptyset$. These voxels located inside white matter are endowed with a way of tracking forward and backward using $\mathcal{F}(M)$ and $\mathcal{B}(M)$. Finally, let $\mathcal{P}$ denote the set of the remaining voxels for which at least one of the two neighbor sets is empty. These voxels are pathological because if one of them is reached during a tracking process, this process is stopped before reaching the set $\mathcal{G}$.

Asking a question about brain connectivity amounts to selecting a subset $\mathcal{G}_{\text {input }}$ of $\mathcal{G}$ and performing a tracking process from this subset. The tracking process simply consists in the construction of a sequence of subsets $\left\{\mathcal{S}_{0}, \mathcal{S}_{1}, \ldots, \mathcal{S}_{n}\right\}$ of the VOI using the following rules (see Fig. 2):

$-\mathcal{S}_{0}=\mathcal{G}_{\text {input }}$

- $\mathcal{S}_{1}=\left\{M \mid \exists M_{-1} \in \mathcal{S}_{0}\right.$ with $M \in \mathcal{F}\left(M_{-1}\right)$ or $\left.M \in \mathcal{B}\left(M_{-1}\right)\right\}$;

- for each $i \in\{2,3, \ldots, n\}, \mathcal{S}_{i}=\left\{M \mid \exists M_{-1} \in \mathcal{S}_{i-1}\right.$ and $\exists M_{-2} \in \mathcal{S}_{i-2}$ with $\left(M \in \mathcal{F}\left(M_{-1}\right)\right.$ and $\left.M_{-2} \in \mathcal{B}\left(M_{-1}\right)\right)$ or $\left(M \in \mathcal{B}\left(M_{-1}\right)\right.$ and $M_{-2} \in$ $\left.\mathcal{F}\left(M_{-1}\right)\right\}$.

The third rule assures that the tracking is always performed in the same direction along the underlying fascicles. Once this sequence has been computed, the answer 


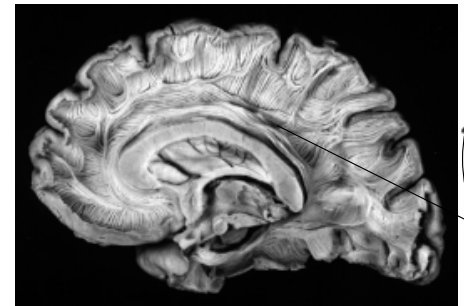

a

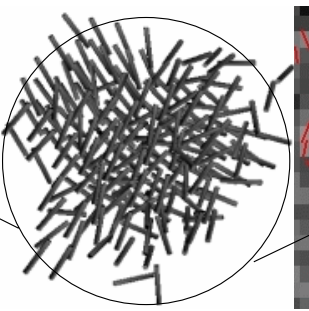

$\mathrm{c}$

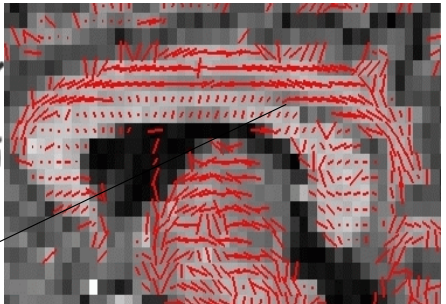

b

Fig. 3. geometry of white matter fascicles; (a) dissection of a human brain (The Virtual Hospital); (b) sagittal slice of anatomical MRI with projection of directions $\boldsymbol{d}(M)$; (c) example of noisy DTI region; each cylinder represents the direction $\boldsymbol{d}(M)$.

to the brain connectivity question is the new subset $\mathcal{G}_{\text {output }}$ of $\mathcal{G}$ defined by:

$$
\mathcal{G}_{\text {output }}=\mathcal{G} \cap \bigcup_{i \in\{1,2, \ldots, n\}} \mathcal{S}_{i}
$$

More sophisticated questions can be answered using the same kind of ideas.

\subsection{Direction Map Regularization}

During the design of the previous tracking method, potential pathological configurations which could perturb the inference of the brain connectivity have been highlighted. In fact, with a noisy direction map, such configurations are bound to be very usual. Indeed, the simple tracking approach introduced above can not tolerate even a one voxel gap in the middle of a fascicle. Unfortunately, DTI contains noise. Moreover, the low resolution of DT images relatively to the usual fiber bundle diameters leads to an important partial volume effect. When a voxel includes several fiber directions, the tensor is very difficult to interpret. In such situations, the main eigen vector can follow a "mean direction" largely different from the directions of the underlying fascicles.

In order to overcome the difficulties induced by noise and partial volume effect, a first idea consists in developing a tracking algorithm dealing with potential gaps using longer links. In fact such a solution seems rather difficult to design in a consistent way. Indeed, the general tracking problem turns out to be relatively "ill-posed", which means that two different diffusion tensor acquisitions of the same brain could lead to two highly different fascicle sets because of noise.

In fact, the tracking difficulties are not induced by the tracking algorithm proposed in the first part of this paper, but by the noisy nature of the direction map made up by the tensor first eigenvectors. Therefore, this direction map has to be restored before applying the tracking. We have recently introduced a class of Markovian models dedicated to such a restoration [6]. In this paper, we focus on one specific model of this class which appears especially adapted to the tracking problem.

The geometry of white matter illustrated by Fig 3. is highly similar to the geometry of spaghetti plates. This analogy between fascicles and spaghetti will 
help us to introduce the mathematical model underlying the construction of the regularized direction map. Let us consider a single spaghetti. Before any cooking, this spaghetti can be considered as a straight line. Put in hot water, the spaghetti becomes a bended curve. The highest the water temperature is, the highest is the spaghetti curvature. A simple way to assess the cooking effect on the spaghetti geometry would consist in integrating the curvature to get some kind of spaghetti bending energy $E$ :

$$
E(\text { spaghett } i)=\int_{0}^{\text {length }} \frac{\mathcal{K}}{2} c^{2}(s) d s ;
$$

where $s$ is simply a curvilinear abcisse along the spaghetti, $c(s)$ is the spaghetti curvature at abcisse $s$ and $\mathcal{K}$ is the stress rigidity. This energy, well known in chemistry as Kratky-Porod model of semi-flexible polymeres [7], can be extended in a straighforward way to a whole spaghetti plate. The fascicle tracking algorithm introduced previously is searching locally for the fascicle with the lowest curvature. This local point of view is not robust to noise in the direction map. This weakness can be overcome if a global point of view is chosen: searching for the fascicle set with the lowest energy of the equivalent spaghetti plate.

The computation of the regularized direction map can be classically interpreted in a Bayesian framework. The optimal direction map $D_{\text {opt }}$ has to maximize the a posteriori probability $p(D / T)$, where $T$ denotes the diffusion tensor data and $D$ denotes a random field which realization scope covers all possible direction maps.

\subsection{Markovian Model}

The realizations of the random variables $\boldsymbol{d}(M)$ of the field $D$ are unitary vectors with any $3 \mathrm{D}$ direction. Let us consider a voxel $M$ of the VOI. This voxel has to belong to one fascicle. $\boldsymbol{d}(M)$ is the putative local direction of this fascicle for a realization of the field. Since fascicles can not end up inside white matter, we have to find neighboring voxels, forwards and backwards, with similar fascicle directions (or perhaps the boundary of white matter). Moreover, this property is sufficient to define the whole geometry of a plausible fascicle set. In the next section, we will propose a Markovian model whose deep local minima correspond to fascicle sets with low curvatures, or spaghetti plates with low energy. Because of this analogy, we will refer to this model as the spaghetti plate model.

The optimal direction map $D_{\text {opt }}$ is a trade-off between the measured tensor data and the a priori knowledge on the low curvature of fascicles. The Bayesian framework leads to the definition of $D_{o p t}$ as the global minimum of the energy $U(D)$ :

$$
U(D)=U_{S}(D)+\alpha U_{T}(D)=\sum_{M} V_{S}(M, D)+\alpha \sum_{M} V_{T}(M, D)
$$

where $U_{S}(D)$ is the regularizing energy related to the analogous spaghetti plate and $U_{T}(D)$ is a quadratic distance to the maximal potential diffusion along spaghetti of the direction map. The constant $\alpha$ allows us to weight the influence 
of the a priori knowledge. The potentials $V_{S}(M, D)$, which are defined on interaction cliques made up by the 26-neighborhood, are inspired by the spaghetti bending energy introduced above (see Eq. 1 and 4):

$$
V_{S}(M, D)=s\left(M, f_{90}(M)\right)+s\left(M, b_{90}(M)\right)
$$

where $f_{90}(M)$ and $b_{90}(M)$ are best linked neighbors related to half-cones with $\beta=90^{\circ}$ aperture angle, covering the whole 26-neighborhood. The potentials $V_{T}(M, D)$, which are constructed from the tensor dot product, measure the discrepancy between diffusion in the direction $\boldsymbol{d}(M)$ and the diffusion in the direction of the tensor first eigenvector $\boldsymbol{e}_{\mathbf{1}}(M)$ :

$$
V_{T}(M, D)=\left(\frac{\boldsymbol{d}(M)^{t} T(M) \boldsymbol{d}(M)-\boldsymbol{e}_{\mathbf{1}}(M)^{t} T(M) \boldsymbol{e}_{\mathbf{1}}(M)}{\|T(M)\|}\right)^{2}
$$

The discrepancy is normalized by the tensor norm [8] in order to get rid of all diffusion-based information apart from anisotropy.

\section{Results}

The state space of the random variables $\boldsymbol{d}(M)$ has been descretized in 162 uniformly distributed directions. A deterministic ICM like algorithm is used to get the $U(D)$ local minimum nearest to the $\boldsymbol{e}_{\mathbf{1}}(M)$ direction map. Regularization has been performed with eleven different values of the weighting parameter $\alpha$. Then, in order to study the influence of regularization on the fascicle set geometry, several subsets of points have been defined from the construction of $\mathcal{F}_{45}(M)$ and $\mathcal{B}_{45}(M)$ extended neighborhoods. It should be noted that the $90^{\circ}$ cone aperture is only used during regularization in order to penalize the worst configurations. In return, during a tracking operation, the aperture angle is largely reduced in order to forbid anatomically meaningless links. The evolution relatively to $\alpha$ of the cardinals of four specific sets of points has been studied (see Fig. 4):

Fascicle nodes: sites endowed with exactly one forward neighbor and one backward neighbor $(\mathcal{B}(M)=\{b(M)\}$ and $\mathcal{F}(M)=\{f(M)\})$;

Junctions: sites related to the merge (or split) of several fascicles made up of points of the previous type $(\operatorname{cardinal}(\mathcal{B}(M)) \times \operatorname{cardinal}(\mathcal{F}(M))>1)$;

Gate to gray matter: sites leading to gray matter $\left(N_{f}(M)=\emptyset\right.$ or $N_{b}(M)=$ $\emptyset)$;

Pathological sites: sites endowed with forward (or backward) basic neighbors, but no forward (or backward) symmetrical neighbors, i.e. pathological sites $\left(\left(N_{f}^{s}(M)=\emptyset\right.\right.$ and $\left.N_{f}(M) \neq \emptyset\right)$ or $\left(N_{b}^{s}(M)=\emptyset\right.$ and $\left.\left.N_{b}(M) \neq \emptyset\right)\right)$.

Fig. 4 presents the evolution from no regularization (right asymptotes) to high regularization (left). First, the number of pathological sites (see Fig. 4.3) decreases dramatically with the regularization which demonstrates the efficiency of the model. Second, the regularization leads to a decrease of the number of junctions and to a dramatic increase of the number of simple fascicle nodes. This effect is mainly due to a reorganization of the fascicles inside larger bundles 

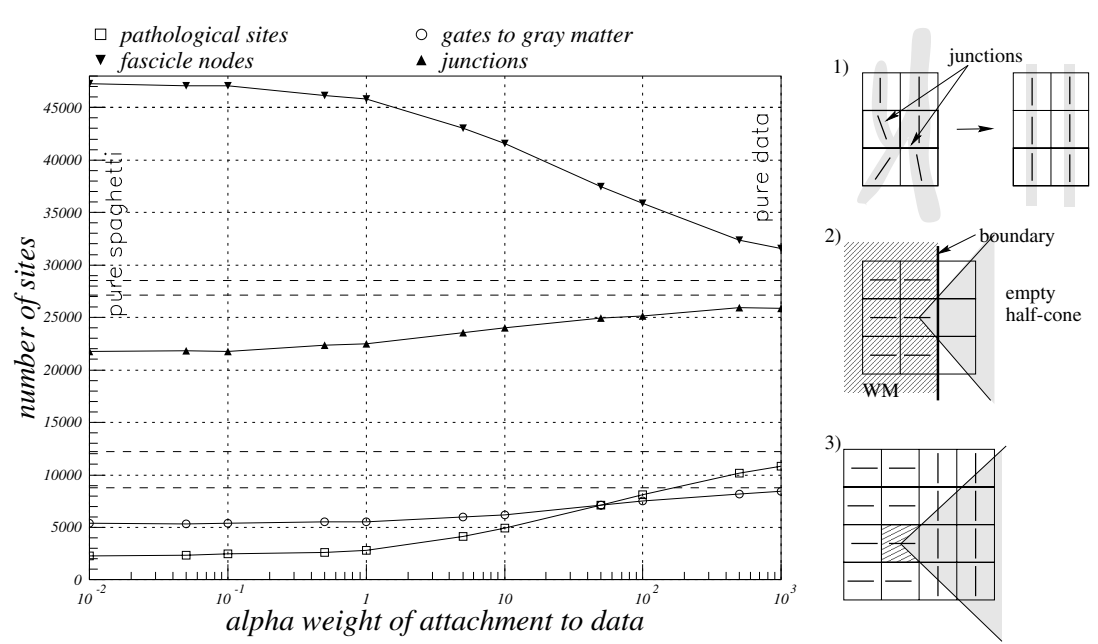

Fig. 4. (left) evolution of the numbers of four different types of configurations; (right) 1) fascicle mixing inside a bundle before regularization versus a bunch of aligned fascicles after regularization; 2) sites of white matter leading to gray matter; 3) pathological site without tracking possibility.

(see Fig. 4.1) which corresponds to the usual underlying anatomical reality. Indeed, the chronotopic establishment of the connections leads to topographically ordered bundles [9]. Hence, the large bundles are endowed with somatotopic organizations, which means that different parts of the bundle section include axons connecting different brain areas. Finally, the number of sites leading to gray matter decreases slightly which is related to the fact that with a $90^{\circ}$ cone aperture during the regularization, only convex areas of the boundary (see Fig. 4.2), namely cortical gyri, are not penalized. While, these areas are the main cortical connexion locations, this effect call for refinements of the regularizing model. All the curve evolutions reach limits beyond which no more topological effect is observed on the fascicle set. This observation suggests that the weight $\alpha=1$ is a reasonable trade-off between regularization and attachment to the data which has been used for further experiments.

In order to illustrate the impact of the regularization on the fiber tracking procedure in a more visual way, we have applied the idea exposed in Fig. 2 with two different inputs (see Fig. 5), one in the motor area (yellow), the other one in the frontal lobe (green). One additional threshold parameter has been added to the tracking process in order to give the user the possibility to focus on fascicles endowed with lower maximal curvature than the one allowed by the tracking cone aperture $\left(\beta=45^{\circ}\right)$ : all best neighbors which do not verify $s(M, f(M))<\gamma^{2}$ are suppressed. In the following $\gamma$ has been fixed to $20^{\circ}$. Fig. 5 presents the tracking results before and after regularization. Two kinds of problems occur before regularization. For the yellow input, in spite of the low threshold, the tracking invades the whole bundles and follows very unplausible trajectories. For the green input, the tracking stops immediately in the middle of white matter while with a higher threshold, the same problem as with the yellow input occurs. 


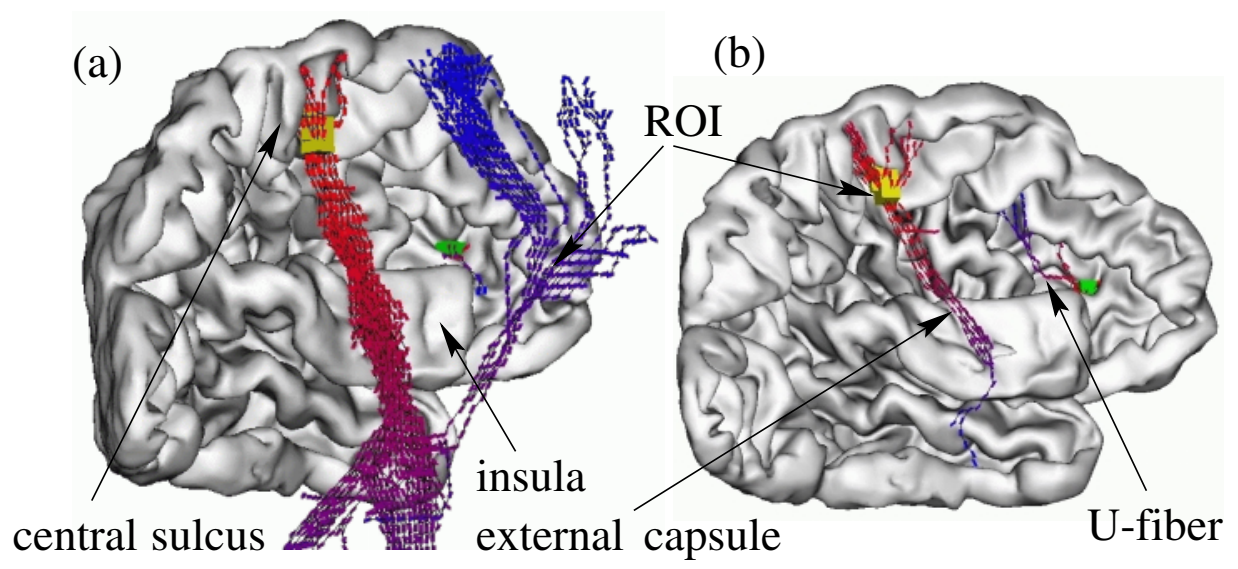

Fig. 5. tracking of fascicles from 2 different inputs [motor area and frontal area]; (a) before regularization; (b) after regularization.

In return, after regularization, the tracking yields much more plausible results, even if a real anatomical validation is currently not available.

\section{Conclusion}

This paper outlines the opening of a new research domain for the image analysis community. Indeed, dealing with tensor images calls for the development of completely new algorithms. This direction of research could lead to the first method giving access in vivo to the human brain connectivity, Such a method would have a great impact both on brain mapping and on pathological studies. Indeed, it should be noted that the connectivity of the human brain and especially of the human cortex is still relatively ill-defined. One of the challenge to take up rapidly is the design of reliable validation approaches using for instance animals and standard anatomical methods.

\section{References}

1. M.P. Young, J.W. Scannell, and G. Burns. The Analysis of Cortical Connectivity. Neuroscience Intelligence Unit. Springer Verlag, 1995. 453

2. D.K. Jones, A.Simmons, S.C.R. Williams, and M.A. Horsfield. Non-Invasive Assessment of Structural Connectivity in White Matter by Diffusion Tensor MRI. In Proceedings of the 6th ISMRM, Sydney, vol. 1, 1998, pp. 531.454

3. J.-F. Mangin, V. Frouin, I. Bloch, J. Régis, and J. Lopez-Krahe. From 3D Magnetic Resonance Images to Structural Representations of the Cortex Topography using Topology Preserving Deformations. Journal of Mathematical Imaging and Vision, vol. 5, 1995, pp. 297-318. 454

4. P.J. Basser, J. Mattiello, and D. LeBihan. Estimation of the Effective Self-Diffusion Tensor from the NMR Spin Echo. Journal of Magnetic Resonance, no. 103, 1994, pp. 247-254. 454 
5. P.J. Basser. Fiber-Tractography via Diffusion Tensor MRI (DT-MRI). In Proceedings of the 6th ISMRM, Sydney, vol. 2, 1998, pp. 1226. 454

6. C. Poupon et al. Regularization of MR Diffusion Tensor Maps for Tracking Brain White Matter Bundles. LNCS 1496, MICCAI'98, Springer-Verlag, MIT Boston, October 1998, pp. 489-498. 458

7. P.M. Chaikin and T.C. Lubensky. Principles of Condensed Matter Physics. Cambridge University Press, 1995. 459

8. P.J. Basser and C. Pierpaoli. Microstructural and Physiological Features of Tissues Elucidated by Quantitative-Diffusion-Tensor MRI. Journal of Magnetic Resonance, no. 111, 1996, pp. 209-219. 460

9. Z. Molnár Development of thalamocortical connections. Springer Verlag, 1998. 461 